\title{
Antioxidant and antimicrobial activities of various propolis samples collected from different regions of Morocco
}

\section{Soumaya Touzani ${ }^{1}$, Nawal El Menyiy ${ }^{1}$, Bratko Filipic ${ }^{2}$, Ilham Elarabi ${ }^{1}$, Noori Al-Waili ${ }^{3}$, Badiaa Lyoussi ${ }^{1}$}

${ }^{I}$ Laboratory of Physiology, Pharmacology \& Environmental Health, Faculty of science, University Sidi Mohamed Ben Abdellah, Fez, Morocco, University Sidi Mohamed Ben Abdallah, Morocco, ${ }^{2}$ Institute for Microbiology and Immunology, Medical Faculty of Ljubljana, University of Ljubljana, Slovenia, ${ }^{3}$ New York Medical Care for Nephrology, Richmond hill, NY, USA

Propolis is a complex natural product produce by honey bees and its composition and antioxidant activity depend on plant origin, season, and area of collection. The present study was performed on various propolis samples collected from different Moroccan regions to assess their antioxidant content and antioxidant and antimicrobial activities. Antioxidant activity was assessed by analyzing hydroalcoholic extracts of propolis by DPPH, ABTS and ferric reducing power assays as well as determining the total phenolic and flavonoid contents. The antimicrobial activity was assessed against different bacterial species and expressed as the minimal inhibitory concentration. The results showed that the antioxidant and antimicrobial activities of different propolis samples are different and depend on the botanical and geographic origin of propolis. Further studies are needed to find the most potent propolis samples that are suitable to be tested in clinical research 